joining its end points. This is possible by virtue of Lemma 1 . Let $\mathcal{f}$ be a second neighborhood of $E_{0}$ interior to $\mathcal{F}^{\prime}$ such that every admissible arc $C$ in $\mathcal{Z}$ joining the points 1 and 2 and satisfying equations (2) cuts the hyperplanes $x=t_{s}$ in points $\left(t_{s}, b_{i s}\right)$ whose $y$-coordinates $b_{i s}$ determine an extremal $E_{b}$ of the family (7) lying in $\mathcal{F}^{\prime}$. By Lemma 1 we have $I_{\lambda}(C) \geqq I_{\lambda}\left(E_{b}\right)$, the multipliers $\lambda_{\alpha}$ being those belonging to $E_{b}$. But since the arcs $C$ and $E_{b}$ satisfy equations (2), this implies that

$$
I_{\lambda}(C)-I_{\lambda}(E)=J(C)-J\left(E_{b}\right) \geqq 0,
$$

the equality holding only in case $C \equiv E_{b}$. Diminish $\mathcal{F}$ if necessary so that $J\left(E_{b}\right) \geqq J\left(E_{0}\right)$, as described in Lemma 2 . We then have $J(C)$ $\geqq J\left(E_{b}\right) \geqq J\left(E_{0}\right)$, the equality holding in both cases only in case $C \equiv E_{0}$. This proves the theorem.

The University of Chicago

\title{
A NEW SUMMATION METHOD FOR DIVERGENT SERIES*
}

\section{W. A. MERSMAN}

1. Introduction. The method to be given here is a modification of that due to Euler-Knopp. $\dagger$ For the weighted means of the partial sums we use the binomial coefficients, but instead of beginning with the first we begin with the "central" one, that is with the greatest. Thus the initial terms always receive the greatest weight, as in the Cesàro-Hölder method.

In this paper it is shown (1) that this new method includes the first two Cesàro methods, and (2) that it also includes the first EulerKnopp method; further, (3) the exact range of summability of the geometric series is determined. Finally, an example is given which indicates that this method may be more powerful than all those of Cesàro-Hölder, although this statement has not yet been proved.

2. Definitions and notation. Throughout we consider a series $\sum_{k=0}^{\infty} a_{k}$ and denote by $S_{n}$ the sum of its first $n+1$ terms. We define $\sigma_{n}$ as follows:

$$
\sigma_{n}=\frac{1}{4^{n}} \sum_{k=0}^{n} C_{2 n+1, n-k} S_{k},
$$

where $C_{n, k}$ denotes the ordinary binomial coefficient. If $\sigma_{n}$ approaches

* Presented to the Society, April 11, 1936. See abstract 42-5-139.

$\dagger$ K. Knopp, Mathematische Zeitschrift, vol. 15 (1922), pp. 226-253. 
a limit as $n \rightarrow \infty$, the original series is said to be summable by our new method.

The regularity of this new method need not be proved directly since it follows from Theorem 1 or Theorem 2 below.

3. Relation to Cesàro's method. In this and the following section we shall make use of a theorem due to Silverman* and Toeplitz: $†$

If two sequences $t_{n}$ and $s_{n}$ are related by the equations

$$
t_{n}=\sum_{k=0}^{n} a_{n k} s_{k},
$$

then necessary and sufficient conditions that $t_{n} \rightarrow s$ whenever $s_{n} \rightarrow s$ are that

(a) $\lim _{n \rightarrow \infty} a_{n k}=0$, for any $k$,

(b) $\lim _{n \rightarrow \infty} \sum_{k=0}^{n} a_{n k}=1$,

(c) $\sum_{k=0}^{n}\left|a_{n k}\right|<M$, independent of $n$.

We begin by recalling the definition of the Cesàro method: If

$$
A_{n}^{r}=\sum_{m=0}^{n} C_{r+m-1, m} S_{n-m},
$$

and if

$$
\alpha_{n}^{r}=A_{n}^{r} / C_{r+n, n},
$$

the series is said to be summable $(C, r)$ if $\alpha_{n}{ }^{r}$ approaches a limit as $n \rightarrow \infty, r$ remaining fixed.

Equating coefficients in the identity $\ddagger$

$$
(1-x)^{r} \sum_{n=0}^{\infty} A_{n}^{r} x^{n}=\sum_{n=0}^{\infty} S_{n} x^{n},
$$

we obtain

$$
S_{n}=\sum_{k=0}^{r}(-1)^{k} C_{r, k} C_{r+n-k, r} \alpha_{n-k}^{r},
$$

and substitution in the definition (1) gives the expression for our means as linear functions of Cesàro's, which enables us to apply directly the theorem of Silverman and Toeplitz. We obtain

* Doctor's thesis, University of Missouri Studies, Mathematics, ser. 1 (1913), pp. 1-96.

$\dagger$ Prace Matematyczno-Fizyczne, vol. 22 (1911), pp. 113-119.

$\ddagger$ S. Chapman, Proceedings of the London Mathematical Society, vol. 9 (19101911), pp. 369-409. 


$$
\sigma_{m}=\sum_{j=0}^{m} a_{m j}^{r} \alpha_{j}^{r}
$$

where

$$
a_{m j}^{r}=\frac{1}{4^{m}} \sum_{n=j}^{j+r}(-1)^{n-j} C_{2 m+1, m-n} C_{r, n-j} C_{r+j, j} .
$$

We have now to prove that the matrix $\left\|a_{m j}^{r}\right\|$ satisfies conditions (a), (b), (c), for $r=2$. We shall verify (a) and (b) for any positive $r$, and (c) for $r=2$.

Proof of (a). We need the asymptotic relation

$$
\frac{1}{2^{2 m+1}} C_{2 m+1, m} \rightarrow(m \pi)^{-1 / 2},
$$

which can be proved either by means of Stirling's theorem* or by means of a result due to Knopp. $†$ Hence, from (4),

$$
\begin{aligned}
\left|a_{m j}^{r}\right| & \leqq \frac{1}{4^{m}} C_{r+j, j} \sum_{n=j}^{j+r} C_{2 m+1, m-n} C_{r, n-j} \\
& \leqq \frac{1}{4^{m}} C_{r+j, j} C_{2 m+1, m} \sum_{n=j}^{j+r} C_{r, n-j} \\
& =\frac{1}{4^{m}} C_{r+j, j} C_{2 m+1, m} \cdot 2^{r} \rightarrow 0 .
\end{aligned}
$$

Proof of (b). Again from (4),

$$
\sum_{j=0}^{m} a_{m j}^{r}=\frac{1}{4^{m}} \sum_{j=0}^{m} \sum_{n=j}^{j+r}(-1)^{n-j} C_{2 m+1, m-n} C_{r, n-j} C_{r+j, j} .
$$

Interchanging the order of summation, we obtain the relation

$$
\sum_{j=0}^{m} a_{m j}^{r}=\frac{1}{4^{m}} \sum_{n=0}^{m}(-1)^{n} C_{2 m+1, m-n} \sum_{j=0}^{n}(-1)^{j} C_{1+j, j} C_{r, n-j} .
$$

Now

$$
\sum_{j=0}^{n}(-1)^{j} C_{r+j, j} C_{r, n-j}=(-1)^{n}
$$

as is easily seen by equating coefficients in the identity

* E. C. Titchmarsh, The Theory of Functions, \$1.87.

$\dagger$ K. Knopp, Theory and Application of Infinite Series, p. 384, example 3. 
Thus

$$
(1-x)^{r} \cdot(1-x)^{-r-1}=\sum_{n=0}^{\infty} x^{n}
$$

$$
\sum_{j=0}^{m} a_{m j}^{r}=\frac{1}{4^{m}} \sum_{n=0}^{m} C_{2 m+1, m-n}=1,
$$

since the sum is exactly half the sum $\sum_{n=0}^{2 m+1} C_{2 m+1, n}=2^{2 m+1}$.

Proof of (c) WITH $r=2$. After putting $r=2$ in (4) and writing out all the terms in the sum, we find that

$$
a_{m j}^{2}=\frac{(2 m+1) !(j+1)(j+2)\left(-m+2 j^{2}+6 j+3\right)}{\left[4^{m}(m-j) !(m+j+3) !\right]},
$$

so that $a_{m j}^{2}$ is negative or positive according as

$$
j \leqq k, \quad j \geqq k, \quad k=\left(\frac{m}{2}+\frac{3}{4}\right)^{1 / 2}-\frac{3}{2} .
$$

Hence

$$
\begin{aligned}
\sum_{j=0}^{m}\left|a_{m j}^{2}\right| & =1+\frac{4(2 m+1) !}{2^{2 m+1}} \sum_{j=0}^{k} \frac{(j+1)(j+2)\left(m-2 j^{2}-6 j-3\right)}{(m-j) !(m+j+3) !} \\
& \leqq 1+\frac{4}{2^{2 m+1}} C_{2 m+1, m} \sum_{j=0}^{k} \frac{(j+1)(j+2)\left(m-2 j^{2}-6 j-3\right)}{(m+2)(m+3)} .
\end{aligned}
$$

Now*

$$
\sum_{j=0}^{k} j^{4}=O\left(k^{5}\right)=O\left(m^{5 / 2}\right)
$$

Therefore

$$
\sum_{j=0}^{m}\left|a_{m j}^{2}\right| \leqq 1+\frac{4}{2^{2 m+1}} C_{2 m+1, m} \cdot O\left(m^{1 / 2}\right),
$$

and, by (5), the right-hand member is bounded. This completes our proof.

Thus we have established the following result:

Theorem 1. Any series summable $(C, 2)$ is summable by this new method to the same sum.

4. Relation to the method of Euler-Knopp. The definition of the Euler-Knopp method is as follows. If

* Cf. E. Lucas, Théorie des Nombres, p. 238, §134, formula 4. 


$$
E_{n}=\frac{1}{2^{n}} \sum_{k=0}^{n} C_{n, k} S_{k},
$$

the series is said to be summable $(E, 1)$ if $E_{n}$ approaches a limit as $n \rightarrow \infty$.

As Rey-Pastor* has shown,

$$
S_{n}=\sum_{r=0}^{n}(-1)^{n-r} C_{n, r} 2^{r} E_{r} .
$$

Substitution in (1) gives our means as linear functions of those of Euler-Knopp:

$$
\sigma_{m}=\sum_{r=0}^{m} a_{m r} E_{r}
$$

where

$$
a_{m r}=\frac{1}{4^{m}} \sum_{n=r}^{m}(-1)^{n-r} C_{n, r} C_{2 m+1, m-n} 2^{r} .
$$

Applying the recursion relation

$$
C_{n, k}=C_{n-1, k-1}+C_{n-1, k},
$$

and proceeding by induction, we obtain

$$
a_{m r}=\frac{2^{r}}{4^{m}} C_{2 m-r, m}>0 .
$$

Proof of (a). Use (5) and (10).

Proof of (b) AND (c). Because of (10), (c) follows from (b). Now, from (9),

$$
\sum_{r=0}^{m} a_{m r}=\frac{1}{4^{m}} \sum_{r=0}^{m} \sum_{n=r}^{m}(-1)^{n-r} C_{n, r} C_{2 m+1, m-n} 2^{r} .
$$

Interchanging the order of summation, we have, by (6),

$$
\begin{aligned}
\sum_{r=0}^{m} a_{m r} & =\frac{1}{4^{m}} \sum_{n=0}^{m} C_{2 m+1, m-n} \sum_{r=0}^{n}(-1)^{n-r} C_{n, r} 2^{r} \\
& =\frac{1}{4^{m}} \sum_{n=0}^{m} C_{2 m+1, m-n}(2-1)^{n}=1 .
\end{aligned}
$$

* Publicaciones de la Facultad de las Ciencias Exactas, Físicas, y Naturales de la Universidad de Buenos Aires, (B), no. 12 (1932), pp. 51-222. 
The result we have just established may be expressed as follows:

Theorem 2. Any series summable $(E, 1)$ is summable by this new method to the same sum.

It may be remarked that, since neither $(C, 2)$ nor $(E, 1)$ summation includes the other, this new method is more general than either of them.

5. The geometric series and other examples. Consider the geometric series, with complex variable $z$,

$$
\sum_{k=0}^{\infty} z^{k}, \quad S_{m}=\sum_{k=0}^{m} z^{k}=\frac{1-z^{m+1}}{1-z} .
$$

Then from (1),

$$
\sigma_{n}(z)=\frac{1}{4^{n}} \sum_{m=0}^{n} C_{2 n+1, n-m} \frac{1-z^{m+1}}{1-z},
$$

or

$$
\sigma_{n}(z)=S(z)+R_{n}(z),
$$

where

$$
S(z)=\frac{1}{1-z}, \quad R_{n}(z)=\frac{z}{z-1} \cdot \frac{1}{4^{n}} \sum_{m=0}^{n} C_{2 n+1, n-m} z^{m} .
$$

From the binomial expansion of $\left[(z+1) / 2 z^{1 / 2}\right]^{2 n+1}$ we find that

$$
R_{n}(z)=\frac{2 z^{1 / 2}}{z-1}\left(\frac{z+1}{2 z^{1 / 2}}\right)^{2 n+1}+R_{n}\left(\frac{1}{z}\right) .
$$

Since the geometric series $(11)$ is summable $(C, 1)$ to the sum $S(z)=1 /(1-z)$ and hence, by Theorem 1 , summable by our method to the same sum, it follows that $R_{n}(1 / z) \rightarrow 0,(|z| \geqq 1, z \neq 1)$. Therefore, from (12), (13), (14) we have the following theorem:

THEOREM 3. The geometric series $\sum_{k=0}^{\infty} z^{k}$, z complex, is summable by this new method to the sum $1 /(1-z)$ in, and only in, the interior of the closed curve whose equation is

$$
\left|\frac{z+1}{2 z^{1 / 2}}\right|=1, \quad \text { or } \quad r^{2}+2 r \cos \theta-4 r+1=0 .
$$

In particular, for $z=-x, x$ real and positive, the range of summability is $-1<x<3+2^{3 / 2}$. 
Also, it is easily seen that the point $z=3 / 2+(-7)^{1 / 2} / 2$ lies on the curve (15); that is, the region of summability extends to the right of the line $R(z)=1$. This shows that no Euler-Knopp method* of any order can include this new method. The same is true of the Cesàro methods, since the region of $(C, r)$ summability of the geometric series is $|z| \leqq 1, z \neq 1$, no matter how large $r$ may be.

We conclude with the example mentioned in the introduction. Consider the sequence

$$
S_{n}=(-1)^{n} C_{n+r, r},
$$

$r$ fixed. From the identity (2) and the Maclaurin expansion of $(1-x)^{-r-1}$ we find (in the notation of $\$ 3$ ) that

$$
A_{2 n}^{r}=-A_{2 n+1}^{r}=C_{n+r, r} .
$$

Hence $\alpha_{n}{ }^{r}$ oscillates between $1 / 2^{r}$ and $-1 / 2^{r}$, so that the sequence (16) is not limitable $(C, r)$. But from (17),

$$
A_{2 n}^{r+1}=C_{n+r, r}, \quad A_{2 n+1}^{r+1}=0,
$$

so that the sequence (16) is limitable $(C, r+1)$ to zero.

We now show that, for any fixed $r,(16)$ is limitable by the new method. In fact, from (1),

$$
\sigma_{n}=\frac{1}{4^{n}} \sum_{m=0}^{n} C_{2 n+1, n-m}(-1)^{m} C_{m+r, r} .
$$

A proof similar to that of (10) above shows that

$$
\sigma_{n}=\frac{1}{4^{n}} C_{2 n-r, n}
$$

and (5) above completes the proof.

The University of California

* K. Knopp, Theory and Application of Infinite Series, p. 510. 